\title{
Alpha-fetoprotein (AFP)-producing epithelial ovarian carcinoma (EOC): a retrospective study of 27 cases
}

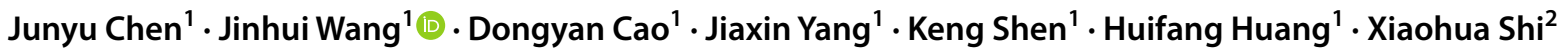

Received: 29 October 2020 / Accepted: 23 February 2021 / Published online: 9 March 2021

(c) The Author(s), under exclusive licence to Springer-Verlag GmbH, DE part of Springer Nature 2021

\begin{abstract}
Objective The aim of the study was to investigate the relative risk factors associated with the prognosis and effective treatments of alpha-fetoprotein (AFP)-producing epithelial ovarian carcinoma (EOC).

Method We presented three cases of AFP-producing EOC and performed a brief review to summarize the clinicopathological features and prognostic factors of 24 cases that have been previously reported. We evaluated the correlations among prognostic and clinical parameters, such as stage, pathology and chemotherapy regimens. In addition, a retrospective review of these 27 cases was conducted, and survival curves were estimated using the Kaplan-Meier method.

Results The patients were aged between 23 and 77 years. The median overall survival was 10 months, and ten (37.04\%) patients died within 18 months. We compared the overall mean survival times of all patients in different stages, and the results suggest that the postoperative pathological staging is hardly correlated with prognosis $(P=0.76)$. There was a correlation between pathology and prognosis $(P=0.0018)$. The mean survival time was longer for patients who had undergone chemotherapy than for those without chemotherapy $(14.88$ vs 0.65 months $)(P<0.0001)$. Moreover, although patients had a good response to the regimens for PEB and TC $(P=0.004)$, there was no significant difference between PEB and TC $(P=0.386)$. Conclusions AFP-producing EOC is uncommon and regarded as an extremely malignant type of tumor. Patients with chemotherapy may have a longer survival time; additionally, PEB and TC may be an optimal selection for this kind of tumor. Further large-scale studies are needed to confirm our findings.
\end{abstract}

Keywords Alpha-fetoprotein (AFP) · Epithelial ovarian carcinoma (EOC) · Yolk sac tumor (YST) · Chemotherapy · Surgery $\cdot$ Prognostic factors

\section{Introduction}

$\alpha$-Fetoprotein (AFP), a fetal serum protein, can be useful as a tumor marker for detecting malignancies such as yolk sac tumors (YSTs), which often occur in young women. AFP is also secreted in the context of other tumors, such as hepatocellular carcinoma and testicular carcinoma [1]. In addition to typical YSTs, epithelial ovarian carcinoma (EOC)

Jinhui Wang

wjhpumch@163.com

1 Department of Obstetrics and Gynecology, Peking Union Medical College Hospital, Chinese Academy of Medical Science and Peking Union Medical College, Beijing 100730, China

2 Department of Pathology, Peking Union Medical College Hospital, Chinese Academy of Medical Science and Peking Union Medical College, Beijing 100730, China with elevated AFP can be easily misdiagnosed due to its infrequent occurrence with a high level of AFP, especially in young women. Moreover, although the likelihood is low, older women can also develop this disease. One article has provided a comprehensive review of AFP-producing ovarian tumors with germ cell differentiation in postmenopausal women [2]. In this review, histopathological findings varied as follows: endometrioid adenocarcinoma, mucinous adenocarcinoma, serous adenocarcinoma, clear cell carcinoma and carcinoma of uncertain type.

Such unusual cases challenge the classic histological diagnosis and are difficult to identify due to the low incidence. It has been suggested that AFP-producing EOC occurs in the same age range as EOC but shows aggressive behavior and poor prognosis [3]. The initial symptoms, such as the presence of an abdominal mass, pain or distention, are identical to those of EOC. However, serum AFP levels are not routinely examined in ovarian tumors arising in older 
women. All of these characteristics lead to difficulty in diagnosing the disease.

The prognoses of malignant germ cell tumor patients are usually good. Fertility-sparing surgery is recommended for those desiring fertility preservation, regardless of stage. Postoperative chemotherapy with bleomycin/etoposide/ cisplatin (PEB) for three to four cycles is recommended. Appropriate surgical staging and debulking surgery following systemic chemotherapy (cisplatin-based) is the initial treatment recommendation for EOC patients. To date, only 24 cases have been reported in the literature. The optimal treatment for AFP-producing EOC is still unclear owing to its rarity. Therefore, accurate diagnosis and treatment need to be conducted effectively. The purpose of this study was to investigate the relevant risk factors for the outcome of AFPproducing EOC according to clinicopathological features and determine effective treatments for this disease.

\section{Method}

Herein, we present three cases of AFP-producing EOC in Peking Union Medical College Hospital: one was a 77-yearold postmenopausal woman with high-grade serous carcinoma, International Federation of Gynecology and Obstetrics (FIGO) IIIC, the second was a 29-year-old woman with poorly differentiated carcinoma, FIGO IIIC, and the third was a 56-year-old postmenopausal woman with poorly differentiated carcinoma, FIGO IIIA. We conducted a literature review and evaluated the clinicopathological features and prognostic factors using published cases of AFP-producing EOC.

\section{Literature search strategy}

We screened for potentially eligible titles, using combinations of the following searching strategy: "(Ovarian Neoplasms[MeSH Terms]) OR (Neoplasm, Ovarian) OR (Ovarian Neoplasm) OR (Ovary Neoplasms) OR (Neoplasm, Ovary) OR (Neoplasms, Ovary) OR (Ovary Neoplasm) OR (Neoplasms, Ovarian) OR (Ovary Cancer) OR (Cancer, Ovary) OR (Cancers, Ovary) OR (Ovary Cancers) OR (Ovarian Cancer) OR (Cancer, Ovarian) OR (Cancers, Ovarian) OR (Ovarian Cancers) OR (Cancer of Ovary) OR (Cancer of the Ovary" OR) "(Carcinoma, Ovarian Epithelial[MeSH Terms]) OR (Carcinomas, Ovarian Epithelial) OR (Epithelial Carcinoma, Ovarian) OR (Epithelial Carcinomas, Ovarian) OR (Ovarian Epithelial Carcinomas) OR (Epithelial Ovarian Cancer) OR (Ovarian Epithelial Cancer) OR (Cancer, Ovarian Epithelial) OR (Cancers, Ovarian Epithelial) OR (Epithelial Cancer, Ovarian) OR (Epithelial Cancers, Ovarian) OR (Ovarian Epithelial Cancers) OR (Ovarian Cancer, Epithelial) OR
(Cancer, Epithelial Ovarian) OR (Cancers, Epithelial Ovarian) OR (Epithelial Ovarian Cancers) OR (Ovarian Cancers, Epithelial) OR (Ovarian Epithelial Carcinoma) OR (Epithelial Ovarian Carcinoma) OR (Carcinoma, Epithelial Ovarian) OR (Carcinomas, Epithelial Ovarian) OR (Epithelial Ovarian Carcinomas) OR (Ovarian Carcinoma, Epithelial) OR (Ovarian Carcinomas, Epithelial)" and "(alphaFetoproteins[MeSH Terms]) OR (alpha Fetoproteins) OR (alpha-Fetoprotein) OR (alpha Fetoprotein)" in the PubMed database, ClinicalTrials.gov, China National Knowledge Infrastructure (CNKI) database and Wanfang Med Online. We identified 27 cases with explicit follow-up periods, the data for which are summarized in Table 1 (including our present cases).

\section{Statistical analysis}

Statistical analysis was performed using the $\mathrm{R}$ version 3.5.3 (R Core Team (2020), R Foundation for Statistical Computing, Vienna, Austria). Survival curves were compared using the Kaplan-Meier method. For all statistical tests, the differences were considered statistically significant when the $P$ value was $<0.05$.

\section{Results}

\section{Case presentation}

\section{Case 1}

A 77-year-old, gravida 3, para 3, Chinese woman was admitted to our hospital with a chief complaint of a palpable pelvic mass and abnormal uterine bleeding. Computed tomography (CT) revealed a $10.5 \times 6.9 \mathrm{~cm}$ solid cystic mass occupying the pelvic and abdominal cavity with rich intralesional vascularization. Positron emission tomography-computed tomography (PET-CT) revealed some metastatic lesions in the mesenterium and peritoneum. However, gastroscopy and colonoscopy showed no mass. The serum tumor markers that were elevated were as follows: $\mathrm{AFP}=27,356.0 \mathrm{ng} / \mathrm{mL}$ and $\mathrm{CA} 125=555.8 \mathrm{U} / \mathrm{mL}$. (The patient's attending doctor decided to test AFP; it was her own opinion, not a routine procedure.) The patient received laparoscopic biopsy, and the pathology was interpreted as undifferentiated carcinoma. Tumor debulking, total hysterectomy, bilateral salpingooophorectomy and omentectomy were carried out after two cycles of preoperative adjuvant chemotherapy consisting of taxol and carboplatin (R1). During the procedure, we found an 8-cm-diametric cystic and solid mass on both sides of the ovaries. Metastatic lesions had spread diffusely throughout 
Table 1 Summary of alpha-fetoprotein producing epithelial ovarian cancer

\begin{tabular}{|c|c|c|c|c|c|c|c|c|}
\hline Authors & Age & Stage & Pathology & Serum AFP (ng/mL) & $\begin{array}{l}\text { Serum } \\
\text { CA-125 } \\
(\mathrm{U} / \mathrm{mL})\end{array}$ & $\begin{array}{l}\text { Completion of } \\
\text { surgery }\end{array}$ & Chemotherapy & Follow-up \\
\hline Nogales [3] & 64 & IA & EC & $>300$ & Elevated & Complete & VDC & DOD $14 \mathrm{~m}$ \\
\hline Nogales [3] & 71 & IA & $\mathrm{EC}$ & Elevated & Elevated & Complete & Cisplatin-based & NED $12 \mathrm{~m}$ \\
\hline Nogales [3] & 31 & IIIC & $\mathrm{EC}$ & 7600 & Elevated & Complete & Cisplatin-based & DOD $8 \mathrm{~m}$ \\
\hline Nogales [3] & 40 & IV & EC & 33 & Elevated & Complete & Cisplatin-based & DOD $6 \mathrm{~m}$ \\
\hline Abe [11] & 52 & IA & $\mathrm{EC}$ & 24,518 & 8439 & Complete & $\mathrm{PEB} \rightarrow \mathrm{TC}$ & NED $20 \mathrm{~m}$ \\
\hline Kamoi [7] & 54 & IA & $\mathrm{EC}$ & 13,143 & 170 & Complete & $\mathrm{C} \rightarrow \mathrm{PEB}$ & NED $21 \mathrm{~m}$ \\
\hline Meguro [2] & 58 & IC & $\mathrm{EC}$ & 102,768 & 324.8 & Complete & $\mathrm{TC}$ & NED $12 \mathrm{~m}$ \\
\hline Kane [20] & 23 & IIIC & EC & 2726 (post) & 85 (post) & Complete & $\mathrm{PEB} \rightarrow \mathrm{P}$ & NED $12 \mathrm{~m}$ \\
\hline Horiuchi [21] & 53 & IA & $\mathrm{EC}$ & 2484.3 & 1349 & Complete & Yes & DOD $6 \mathrm{~m}$ \\
\hline $\operatorname{Li}[6]$ & 41 & IIIB & $\mathrm{EC}$ & 5202 & 85.2 & Complete & PEB & NED $8 \mathrm{~m}$ \\
\hline Lawrence [22] & 48 & IA & $\mathrm{EC}$ & 200 & NA & Complete & Yes & NED $24 \mathrm{~m}$ \\
\hline Maida [23] & 53 & IIIC & $\mathrm{CCC}$ & 2759 & 438 & Suboptimal & CAP & NED 4 m \\
\hline Li [24] & 62 & IV & $\mathrm{CCC}$ & $>350$ & 488 & Complete & $\mathrm{PEB} \rightarrow \mathrm{GP}$ & AWD $40 \mathrm{~m}$ \\
\hline Chao [25] & 52 & IIIC & $\mathrm{CCC}$ & 60,721 & 38.1 & Complete & $\mathrm{TC} \rightarrow \mathrm{T}$ & DOD $18 \mathrm{~m}$ \\
\hline Lawrence [22] & 49 & IIIA & $\mathrm{CCC}$ & 300 & NA & Complete & Yes & DOD $15 \mathrm{~m}$ \\
\hline Morimoto [26] & 59 & IIB & $\mathrm{CCC}$ & 1606 & Normal & Complete & $\mathrm{TC}$ & NED $23 \mathrm{~m}$ \\
\hline Takahashi [27] & 54 & IIIC & $\mathrm{CCC}$ & 4159 & Elevated & Complete & $\mathrm{TC}$ & NED $48 \mathrm{~m}$ \\
\hline Konishi [28] & 53 & IIIC & $\begin{array}{l}\text { Mucinous cystadeno- } \\
\text { carcinoma }\end{array}$ & 1120 & 27 & Complete & Yes & DOD $7 \mathrm{~m}$ \\
\hline Nomura [29] & 62 & IIIC & $\begin{array}{l}\text { Mucinous cystadeno- } \\
\text { carcinoma }\end{array}$ & 4130 & NA & Complete & No & DOD $1 \mathrm{~m}$ \\
\hline Arai [30] & 71 & IC & $\begin{array}{l}\text { Mucinous cystadeno- } \\
\text { carcinoma }\end{array}$ & 55.6 & NA & Complete & Cisplatin-based & DOD $7 \mathrm{~m}$ \\
\hline Suzuki [31] & 66 & IIIB & $\begin{array}{l}\text { Serous adenocarci- } \\
\text { noma }\end{array}$ & 17,013 & 360 & Complete & $\mathrm{TC}$ & NED $10 \mathrm{~m}$ \\
\hline Isonishi [32] & 59 & III & $\begin{array}{l}\text { Poorly differentiated } \\
\text { adenocarcinoma }\end{array}$ & 73,687 & 1599 & Complete & PEB & NED $3 \mathrm{~m}$ \\
\hline Nogales [3] & 73 & IIIC & MMMT & 23 & Elevated & Complete & Yes & AWD $2 \mathrm{~m}$ \\
\hline Lawrence [22] & 67 & IIIC & MMMT & $>51,000$ & NA & Complete & No & DOD $10 \mathrm{~d}$ \\
\hline Current report 1 & 77 & IIIC & Serous carcinoma & 27,356 & 555.8 & Suboptimal & $\mathrm{TC}$ & NED $2 \mathrm{~m}$ \\
\hline Current report 2 & 29 & IIIC & $\begin{array}{l}\text { Poorly differentiated } \\
\text { carcinoma }\end{array}$ & 19,754 & 169.7 & Suboptimal & PEB & NED $6 \mathrm{~m}$ \\
\hline Current report 3 & 56 & IIIA & $\begin{array}{l}\text { Poorly differentiated } \\
\text { carcinoma }\end{array}$ & 2820 & 171.2 & Complete & $\mathrm{TC} \rightarrow \mathrm{PEB} \rightarrow \mathrm{PE}$ & AWD $47 \mathrm{~m}$ \\
\hline
\end{tabular}

$E C$ endometrioid carcinoma; $C C C$ clear cell carcinoma; $M M M T$ malignant mixed mesodermal tumor; $N A$ not available; $P E B$ bleomycin/etoposide/cisplatin; $T$ paclitaxel or docetaxel; $C$ carboplatin; $T C$ paclitaxel/carboplatin; $V D C$ vincristine/dactinomycin/cyclophosphamide; $C A P$ cisplatin/pirarubicin hydrochloride/cyclophosphamide; $G P$ gemcitabine/cisplatin; $N E D$ no evidence of disease; $A W D$ alive with disease; $D O D$ died of recurrent disease

the peritoneum, the omentum and the surface of the bladder and rectouterine fossa.

\section{Pathology report}

The pathology of this original surgery was interpreted as high-grade serous carcinoma of both ovaries with the involvement of metastatic lesions on the surface of the omentum, bladder, rectum and pelvic area, partially with a YST component. Grossly, the right and left ovarian tumors were 10 and $8 \mathrm{~cm}$ in diameter, respectively, and the lesions were very fragile with a nodularity like a rotten flesh surface. The cut section showed a gray yellow focus and partial hemorrhage and a necrotic area. There was also a mass of yellowish-gray tissue with a total volume of $5 \times 3 \times 1 \mathrm{~cm}$ on the surface of the rectum. Microscopic findings show highgrade papillary serous carcinoma with yolk sac component. Immunohistochemistry (IHC) was performed to confirm the ultimate histological diagnosis. The tumor cells were positive for AE1/AE3, CK7 and P53, focally positive for AFP 
and SALL-4 and negative for OCT3/4, WT-1, $\alpha$-inhibin, PAX-8, PR and ER $\alpha$. A definite diagnosis of ovarian highgrade serous carcinoma (FIGO stage IIIC) was made based on the clinical presentation, histopathological features and IHC profiles (Fig. 1).

The patient was treated postoperatively with two cycles of chemotherapy consisting of Taxol and carboplatin. After two cycles of chemotherapy, serum AFP decreased to $47.2 \mathrm{ng} /$ $\mathrm{mL}$, and CA125 decreased to $88.7 \mathrm{U} / \mathrm{mL}$. No signs of clinical or radiological evidence of disease recurrence were found at follow-up examinations during the first 2 months after the operation.

\section{Case 2}

A 29-year-old gravida 2, para 1, Chinese woman consulted our hospital with a complaint of lower abdominal discomfort. A recent ultrasound test revealed two $5 \times 3 \mathrm{~cm}$ solid cystic masses occupying both sides of the ovaries with rich intralesional vascularization. Her CA125 levels were elevated to $169.7 \mathrm{U} / \mathrm{mL}$. AFP was also performed to evaluate whether she had ovarian germ cell tumor, such as yolk sac tumor, and was elevated to $19,754.0 \mathrm{ng} / \mathrm{mL}$. CT revealed multiple metastatic lesions in the peritoneum. A right salpingo-oophorectomy was performed, and intraoperative frozen section consultation showed an ovarian malignant tumor (the possibility of YST cannot be excluded); therefore, right salpingo-oophorectomy and omentectomy along with the removal of pelvic metastases were conducted. The large mass arising from the ovary was removed, along with the involvement on the surface of peritoneum, omentum, sigmoid colon, rectum and stomach. However, some disseminated lesions over $1 \mathrm{~cm}$ on the uterorectal fossa and stomach remained.

\section{Pathology report}

The pathology was interpreted as poorly differentiated carcinoma with metastatic lesion involvement on the surface of the omentum, bladder, rectum and sigmoid colon, partially with yolk sac differentiation, FIGO IIIC. Grossly, the right ovarian tumor was $5.3 \times 3.5 \times 3.5 \mathrm{~cm}$ in diameter and
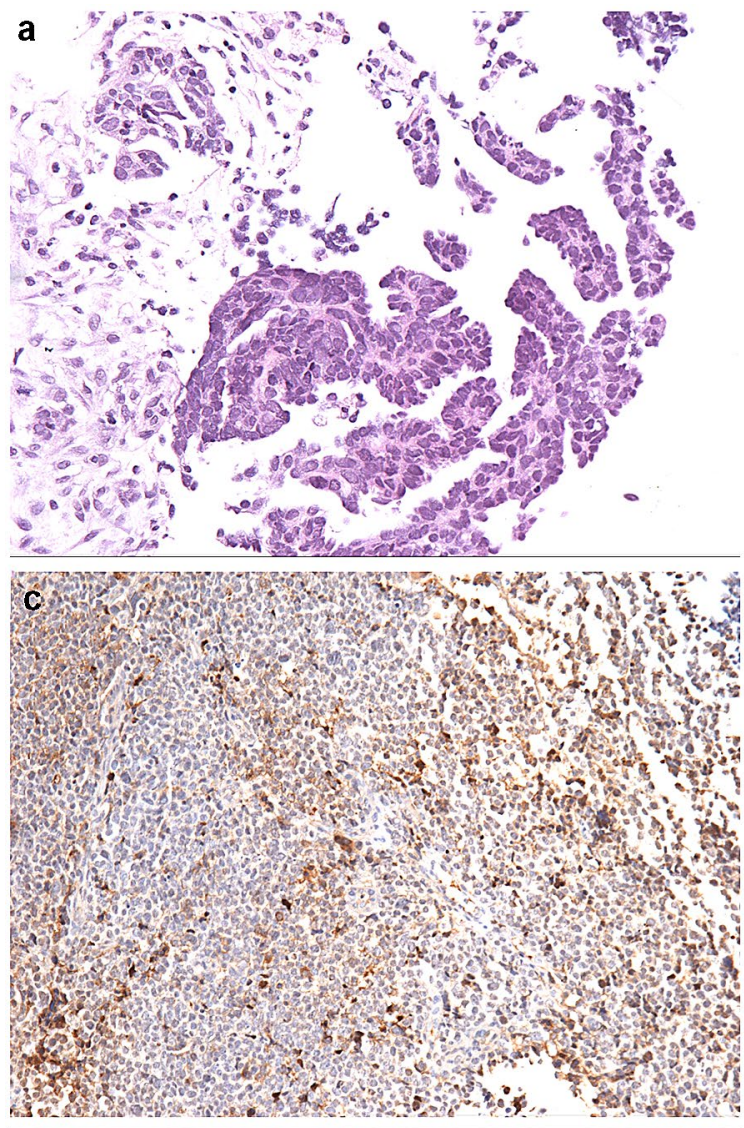

Fig. 1 Histologic findings of the ovarian tumor in case 1. a Highgrade papillary serous carcinoma. HE $100 \times$, b Solid growth pattern of the tumor in the yolk sac differentiation area. HE 100×, c AFP
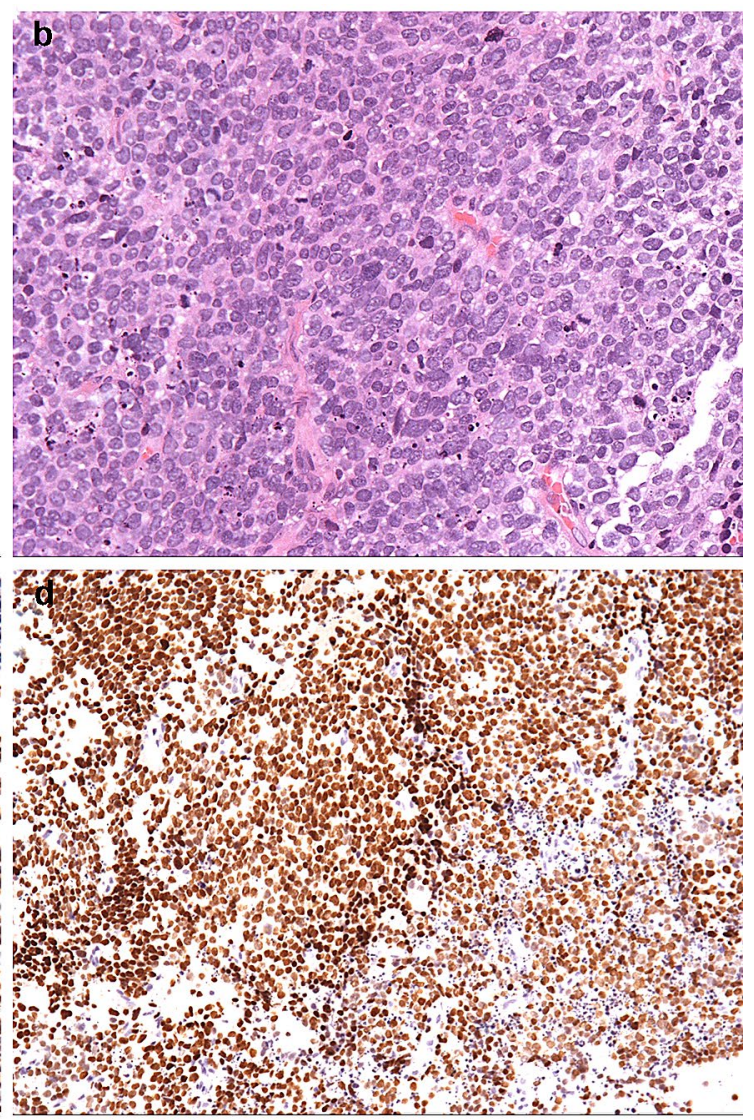

immunohistochemical staining is positive in the solid growth area. $100 \times, \mathbf{d}$ SALL4 immunohistochemical staining is positive in the solid growth area. $100 \times$ 
had a fibrous capsule which was partly ruptured. The cut surface was mostly solid and gray-white with focal hemorrhage and necrosis. Microscopically, pseudo-glandular growth pattern and solid growth pattern were found in the tumor. The nuclear is sever atypical, with prominent nucleoli. The cytoplasm is clear and eosinophilic. Atypical mitosis can be seen in the upper right quadrant. Immunohistochemically, the tumor cells were positive for AFP and focally positive for CK7 and EMA. No apparent expression of OCT3/4, SALL-4, Sox-2 or PAX-8 was noted (Fig. 2).

Because initially, the tumor was suspected to have yolk sac differentiation, the patient was treated postoperatively with one cycle of chemotherapy consisting of bleomycin, etoposide and cisplatin. After a cycle of chemotherapy, serum AFP decreased to $705.7 \mathrm{ng} / \mathrm{mL}$, and serum CA125 decreased to the normal value. To confirm the diagnosis, the patient underwent gastroscopy. A biopsy revealed a poorly differentiated carcinoma of the stomach. We recommended a recheck of the two pathology results to confirm the origin of the tumor. However, the patient refused to do so and interrupted treatment herself due to COVID-19 pandemic. Then, she lost contact six months later.

\section{Case 3}

A 56-year-old, gravida 3, para 2, Chinese woman presented with a complaint of lower abdominal pain. Before visiting our hospital, she was found to have a pelvic mass on pelvic examination and ultrasound as well as high level of serum tumor markers, such as AFP and CA125, in other hospital. A recent ultrasound examination showed a $10.3 \times 9.4 \times 7.1 \mathrm{~cm}$ mixed echogenic mass with an obvious blood flow signal, but ovaries were not detected. We retested the tumor markers, and the results were as follows: serum $\mathrm{AFP}=2820 \mathrm{ng} /$ $\mathrm{mL}, \mathrm{CA} 125=171.2 \mathrm{U} / \mathrm{mL}$ and CA199 $=380.9 \mathrm{U} / \mathrm{mL}$. The patient underwent exploratory laparotomy. There was $500 \mathrm{~mL}$ hemorrhagic ascitic fluid in the abdominal cavity, and the left ovary was replaced by a tumor, $12 \mathrm{~cm}$ in size, which was adhered to the rectouterine fossa. No lesions were found in other areas. Most of the tumor was removed, and the fast-frozen pathology result was malignant tumor. Total hysterectomy with bilateral salpingo-oophorectomy, omentectomy, appendectomy and pelvic lymphadenectomy was performed without any residual tumor (R0).
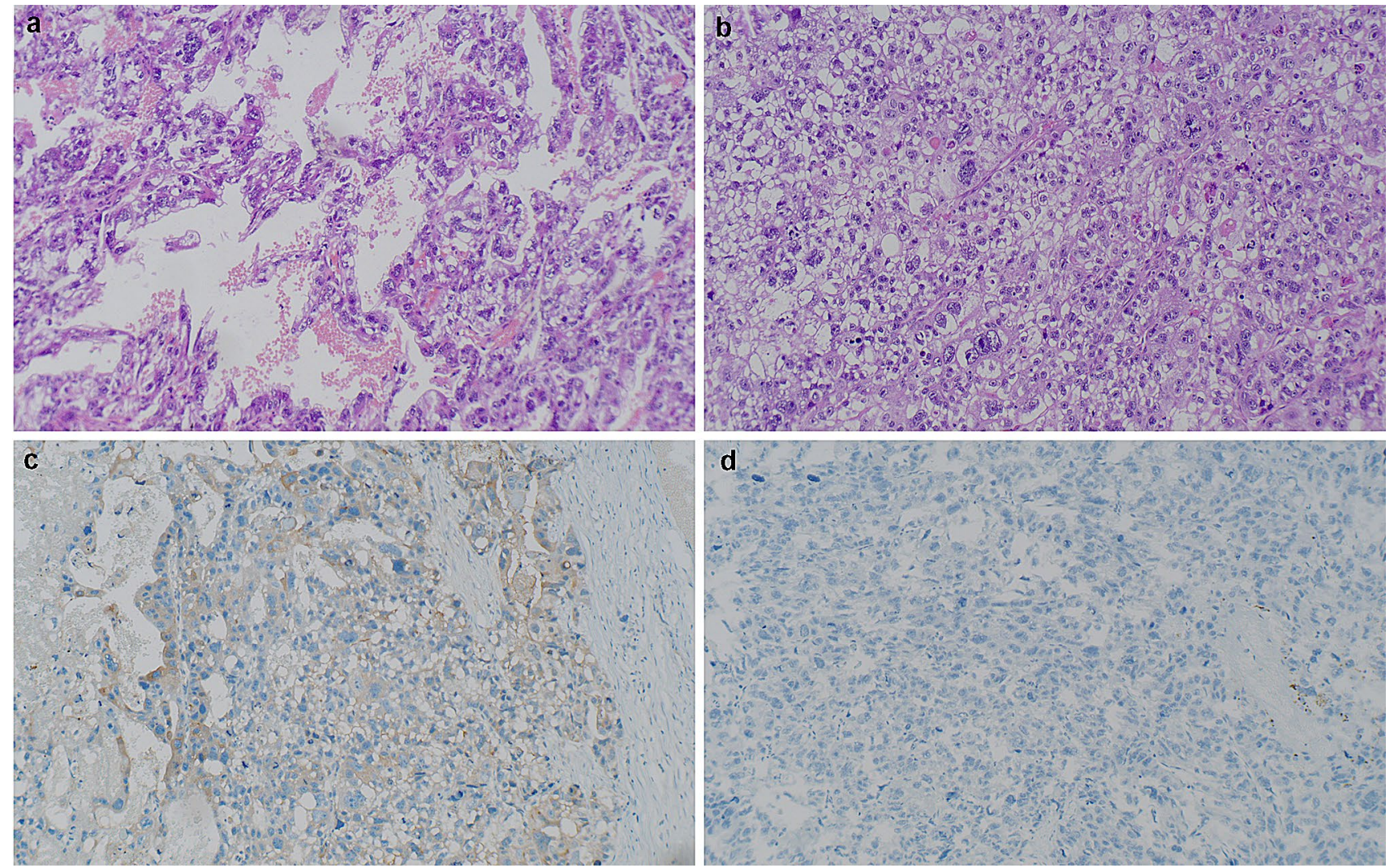

Fig. 2 Histologic findings of the ovarian tumor in case 2. a Pseudoglandular growth pattern. HE $100 \times$, b Solid growth pattern. The nucleus is severely atypical, with prominent nucleoli. The cytoplasm

is clear and eosinophilic. Atypical mitosis can be seen in the upper right quadrant. HE $100 \times$, c AFP is positive. $100 \times$, d SALL-4 is negative. $100 x$ 


\section{Pathology report}

Pathologic examination demonstrated poorly differentiated carcinoma with primary endoderm differentiation, involving the left ovary, left fimbriae tubae and appendix, FIGO IIIA. Gross finding of the samples displayed a left ovarian tumor measuring $11 \times 10 \times 5 \mathrm{~cm}$, whose lesions were very fragile with an irregular surface. The cut surface appeared to extensive rotten fish-like texture with necrosis and hemorrhage. Immunohistochemical staining was performed in order to confirm the histological diagnosis, and the tumor cells were positive for AE1/AE3, AFP, CK7 and EMA. While immunostaining, CA125, SALL-4, ER, PAX-8 and OCT3/4 were negative. Additionally, the positive rate of Ki67 staining was about $80 \%$. A definite diagnosis of poorly differentiated carcinoma of right ovary (FIGO stage IIIA) was made.

The patient was treated postoperatively with six cycles of chemotherapy consisting of taxol and carboplatin. The serum levels of these markers decreased to normal values after one cycle of chemotherapy. The patient had a recurrence four times and received seven cycles of PEB, four cycles of a combination of PE and bevacizumab, 6 months of radiotherapy and 8 months of etoposide. The patient is alive with the disease, and it has been 47 months since the operation.

\section{Clinicopathological features and prognostic factor analysis}

To the best of our knowledge, 27 EOC cases with preoperative serum AFP elevation have been documented so far. The clinical features and histologic findings of these cases are summarized in Table 1 and Fig. 3. These patients were aged from 23 to 77 years, and most of the cases of this type of tumor have occurred in postmenopausal or perimenopausal women. Seventy-four percent of the patients were over 50 years old, but we were aware of five patients who were younger (23-41 years).

Approximately $67 \%$ of the patients in our study presented with advanced-stage disease (18 were FIGO stage III or IV; eight were stage I; and one was stage II). A Kaplan-Meier survival curve of the 27 cases, based on data in the published literature, is shown in Fig. 4a. For all stages, the median overall survival time was 10 months, and the average overall survival time was 13.83 months, while ten $(37.03 \%)$ patients died within 18 months. The survival curves for each stage

a

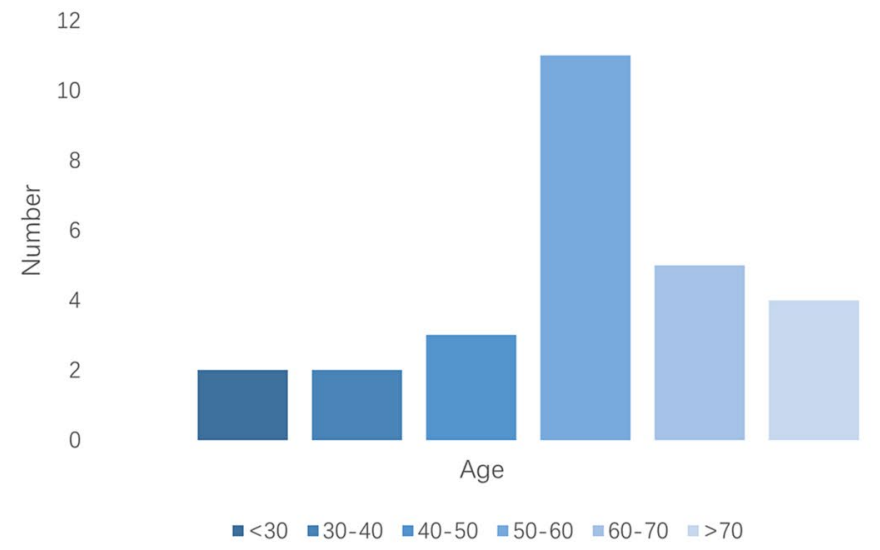

b

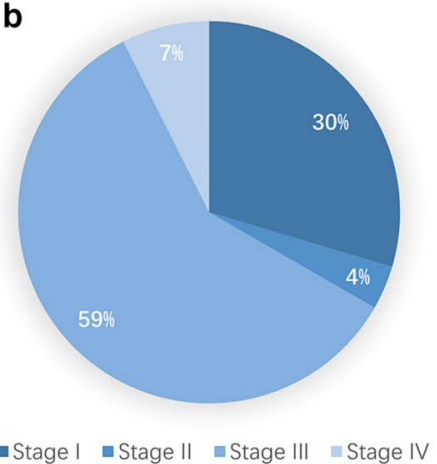

-Stage | $\mid$ Stage II -Stage III $=$ Stage IV c

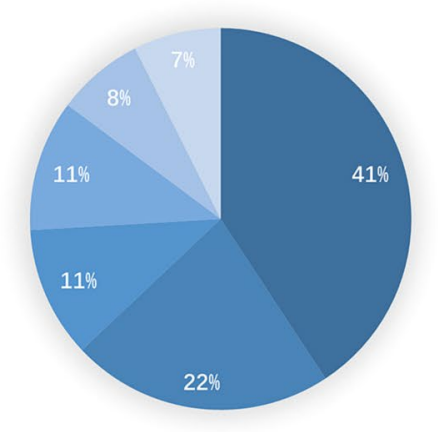

- Endometrioid carcinoma

- Clear cell carcinoma

Mucinous cystadenocarcinoma

= Poorly differentiated carcinoma

= Serous adenocarcinoma

Malignant mixed mesodermal tumors

Fig. 3 Epidemiological information of patients. Distributions of age (a), stage (b) and pathology (c) 

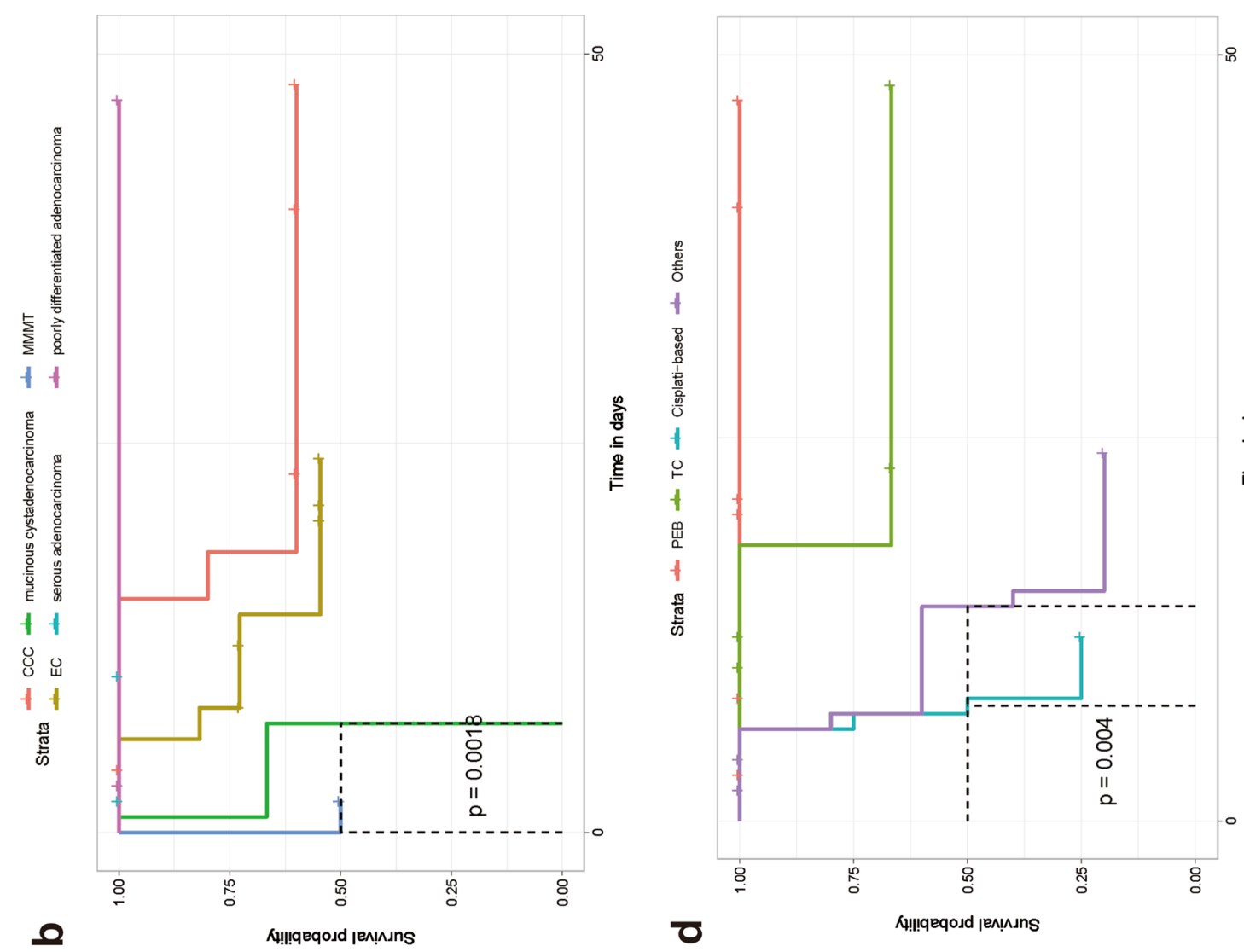

(⿸丆口

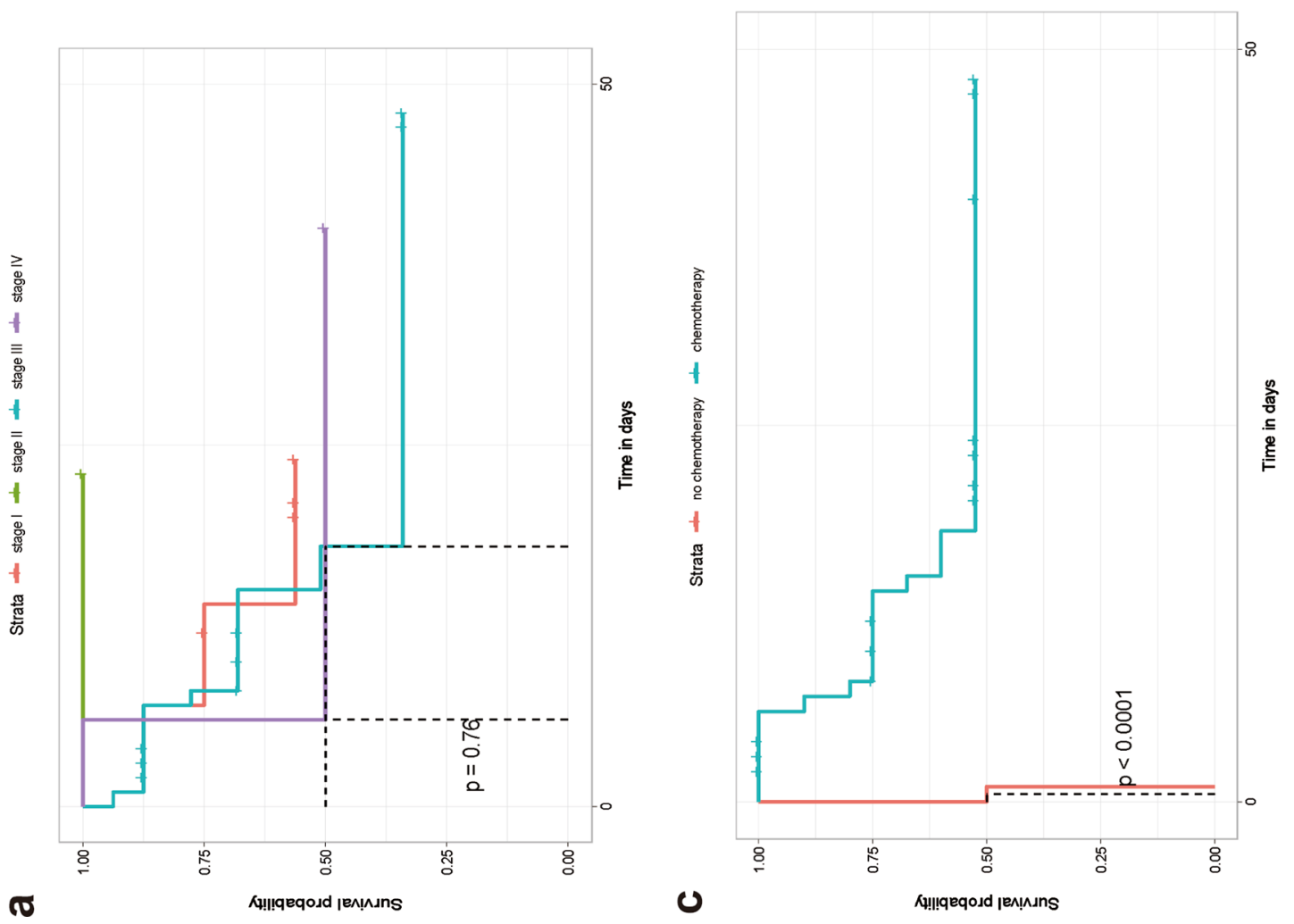

亩

总

उ $\overline{0}$

政

记主

일

e

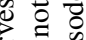

亏 :

ब

等

:

은

宅

츨

要

政

造

बิ

政

可 응

论

产

势

要

용

3 可

这

胥

등

可范

$\sum_{0}$

骆

월

घ 응

तु

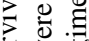

क 3

콜 슬

言

¿

is

跣

웡

웡

政

궁

可 密

$\sum$ 글

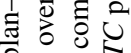

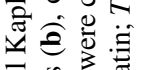

न

20

क न

ज्ञ

o 0 i

$\checkmark$ 羟

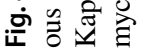


had route near-overlap, which suggested that postoperative pathological staging is hardly correlated with prognosis $(P=0.76)$.

Of the 27 cases, almost all patients had YST components. Endometrioid carcinoma was the most common type, occurring in 11 patients (40.74\%), and six of these were associated with clear cell carcinoma (22.22\%). Mucinous cystadenocarcinoma and poorly differentiated carcinoma occurred in three cases, and serous adenocarcinoma and malignant mixed mesodermal tumors (MMMTs) occurred in two cases. We tried to determine the correlation between pathological classification and prognostic evaluation in these 27 cases and define it in a clearer manner. The results showed that there was a correlation between pathology and prognosis $(P=0.0018)$ (Fig. 4b). Mucinous cystadenocarcinoma seems to have an undesirable prognosis, but we were unable to determine the influence of tumor pathological type on prognosis based on the very small number of cases.

In almost all cases, the patients received total hysterectomy with bilateral salpingo-oophorectomy with or without omentectomy and/or lymph node dissection. Only two patients did not receive chemotherapy; one of them died within 10 days, while the other died within one month. The mean survival time of patients with chemotherapy was 14.85 months, and that of patients without chemotherapy was 0.65 months. There was a firm correlation between chemotherapy and prognosis $(P<0.0001)$, as shown in Fig. 4c. In addition, we analyzed the difference between different regimens (Fig. 4d). Patients had a good response to the regimens for PEB and TC $(P=0.004)$. However, no significant difference between PEB and TC $(P=0.386)$ was found.

\section{Discussion}

AFP, one of the fetal serum proteins in the developing embryo, is synthesized mainly by the liver and yolk sac, and its levels are often increased in malignancies. AFP is well established as a useful diagnostic tumor marker for hepatocellular carcinomas, YST, embryonal carcinoma of the ovary, and some testicular cancers [4, 5]. However, EOCs have rarely been reported to secrete AFP. In this article, we described three cases of AFP-producing ovarian tumors: a 77-year-old postmenopausal woman with high-grade serous carcinoma (FIGO IIIC), a 29-year-old woman with poorly differentiated carcinoma (FIGO IIIC) and a 56-year-old postmenopausal woman with poorly differentiated carcinoma (FIGO IIIA). We also presented a retrospective review of 27 cases and analyzed the relationship between prognostic factors and disease outcome.

In this investigation, we found that most patients diagnosed with this disease were over 50 years old, which means that it often occurs in postmenopausal women in the same age range as EOC. Nevertheless, the age distribution contrasts with the age of patients with typical germ cell tumors, which often occur in adolescents and young adults. However, there were five patients who were younger (23-41 years) in our research. Hence, a young patient with a pelvic mass with a high level of AFP in the hospital was easily diagnosed with a germ cell tumor. In addition, AFP was not routinely examined in ovarian tumors arising in older women. Both of these situations might lead to a misdiagnosis and delayed treatment. Of the 27 patients, $66.67 \%$ had advanced-stage disease. Their disease-free survival times were found to be from 2-48 months depending on the length of follow-up. For all stages, the median overall survival time was 10 months, and the average overall survival time was 13.83 months, while ten $(37.03 \%)$ patients died within 18 months. This observation revealed that postoperative pathological staging was hardly correlated with prognosis $(P=0.76)$.

These AFP-producing EOCs are generally considered to have a poor prognosis, and the optimal approach to management is still unknown. Currently, consisting of paclitaxel and carboplatin is the first-line chemotherapeutic regimen for advanced EOC, and the optimal treatment for germ cell tumors is a combination of bleomycin, etoposide and cisplatin. It was previously postulated that adjuvant therapy for AFP-producing EOCs should include aggressive platinumbased chemotherapy. However, some evidence has shown that patients also have a good response to the PEB regimen [6]. Therefore, it is worth considering whether the patient should be initially treated with chemotherapy that is effective in the context of a malignant GCT and additional cycles of platinum-based chemotherapy for ovarian carcinoma. It is worth noting that Kamoi et al. reported better survival following five cycles of PEB chemotherapy and one cycle of intraperitoneal carboplatin [7]. According to a published paper, almost all patients undergo adjuvant chemotherapy treatment, and PEB and platinum-based chemotherapy account for the majority of cases. In addition, other chemotherapy regimens, such as VDC and CAP, are also applied in some patients. Because of the limited number of reports, there is no consensus on the optimal treatment regimen for this rare tumor type. In this paper, we compared the mean survival times of patients with chemotherapy (14.85 months) and patients without chemotherapy (0.65 months) using a Kaplan-Meier curve. Fortunately, we found a strong correlation between chemotherapy and prognosis $(P<0.0001)$. This revealed that patients with chemotherapy may have a longer survival time. However, only two cases did not receive chemotherapy; thus, we still need a larger sample size to support our results. Moreover, we also analyzed the difference between different regimens. Similar to the former study, patients had a good response to the regimens for PEB and TC, even though there was no difference between these 
two regimens $(P=0.386)$ [2]. This result might provide an option for the treatment of AFP-providing EOCs. Bleomycin is considered an essential component of the PEB regimen. Bleomycin-induced pulmonary toxicity is observed in approximately $10 \%$ of patients treated with PEB, with clinical symptoms such as dry cough, dyspnea, crackles during auscultation, abnormalities on chest radiography and fever [8]. Patients with lung diseases, especially older patients, may not be tolerant of side effects. Furthermore, bleomycin has a lifetime dose limit, which means that patients can receive only a limited cycle of chemotherapy. Under these circumstances, considering the same therapeutic effect of the two regimens, the TC regimen seems to be a better option for treating disease. For treating young patients with AFP-producing EOCs, some people believe it is not necessary to distinguish EOC from YSTs given that PEB is the treatment for both tumors. YST patients are usually sensitive to systemic chemotherapy and have a better prognosis, and fertility-sparing surgery could proceed for those who desire fertility preservation regardless of stage. AFPproducing EOC has a poor prognosis, and a radical approach was considered. Therefore, early and correct diagnosis of AFP-producing EOCs could help doctors choose optimal management.

It has been reported that carcinomas of somatic origin are occasionally associated with elevated serum AFP and positive staining for AFP $[9,10]$. Abe. A suggested that ovarian tumors with a YST component should be considered a rare variant, and the development of extraembryonic germ cell tissue from tumors of somatic origin is a rare event in the female genital tract and occurs mainly in the aerodigestive and urinary tracts [11]. However, four theories, namely the teratoma theory, retrodifferentiation theory, collision theory and neometaplasia theory, have been proposed, and the exact explanation for this biological behavior is not known [7, $12,13]$. In our research, endometrioid carcinoma was the most common type, occurring in $40.74 \%$ of the patients, and the second most common type was clear cell carcinoma (22.22\%). Mucinous cystadenocarcinoma, poorly differentiated carcinoma, serous adenocarcinoma and MMMTs also occurred in several cases. Almost all patients had a YST component. As germ cells are not identified histologically in the ovaries of postmenopausal women, a direct origin of malignant neoplasms from germ cells is highly unlikely at that age. We highly suspected that the rare tumor represents an EOC with aberrant differentiation because it occurs in the same age range as EOC and shows aggressive behavior, but the molecular events involved in this transformation have not yet been elucidated. Our results also showed that there was a correlation between pathology and prognosis $(P=0.0018)$. MMMTs and mucinous cystadenocarcinoma had undesirable prognoses compared to other types, which suggests that we need to be more aggressive in the treatment for these types of tumors. However, we were unable to determine the influence of tumor pathological type based on the very small number of cases.

Immunohistochemical expression of AFP was found in all three cases in our study, which explains the high level of serum AFP. Similar to the previous study, it is speculated that these AFP-positive components might be differentiated into yolk sac components [2]. One study indicated that SALL4, which is a novel oncofetal marker expressed specifically in primitive germ cell tumors, would be of use in discriminating between YSTs and EOCs [14]. Interestingly, in our preliminary study, the expression of SALL4 was detected in the case of high-grade serous carcinoma but not in the cases of poorly differentiated carcinoma. The possible reason is that we found a yolk sac differentiation area in case 1, which may lead to the expression of SALL4. Furthermore, all three cases were positive for CK7, with most studies showing these markers to be negative in YST, suggesting that this kind of tumor has a similarity to conventional ovarian epithelial tumors [15]. In addition, OCT3/4 expression has been reported only in high-grade immature ovarian teratomas [16]. In our study, all three cases were negative for OCT3/4, thus confirming their usefulness in distinguishing between the two components. PAX8, a broad Müllerian-derived tumor marker, is frequently expressed by EOC components but never in YST elements [17, 18]. However, in our study, no apparent expression of PAX8 was noted in these cases. Based on these findings, some "characteristic immunohistochemical markers," including AFP and PAX8, cannot be reliably used to distinguish between YST and AFP-producing EOC. A wide comprehensive panel including SALL 4, OCT3/4 and CK7 may improve the diagnostic accuracy in these cases. However, we still need to find a panel of markers to facilitate the distinction and reduce the misdiagnosis rate.

In conclusion, AFP-producing EOC is uncommon and an extremely malignant type of tumor whose prognosis is poor even if an early diagnosis is made. Serum AFP levels are not routinely examined in patients with ovarian tumors, especially in older women. Additionally, the main concern for young patients with AFP elevation is a YST, which has a better prognosis than AFP-producing EOCs. The pathologist needs to be alert, as small foci of YST with an uncommon papillary-like pattern should not be mistaken for epithelial tumors. To date, there is limited evidence to support an ideal first-line chemotherapy regimen for this tumor type. Complete debulking initial surgery and adjuvant chemotherapy, namely PEB and TC, may be an optimal selection for this kind of tumor. In most cases, the elevation in AFP is often associated with the elevation in CA-125. However, AFP seemed to be more sensitive for tumor follow-up than CA-125 [19]. We can 
efficiently evaluate therapeutic effects by monitoring the level of AFP.

It is highly recommended that a global medical database of AFP-producing EOC be established to collect and analyze interinstitutional clinicopathological data that can further be discussed and shared at oncology conferences. Additionally, to have a clear understanding and improve the management of AFP-producing EOC, more retrospective and prospective clinical studies need to be conducted in the future.

Authors' contributions Jinhui Wang contributed to conceptualization. Junyu Chen and Xiaohua Shi contributed to data curation. Junyu Chen and Jinhui Wang performed formal analysis. Junyu Chen contributed software. Junyu Chen and Jinhui Wang were involved in writingoriginal draft. Dongyan Cao, Jiaxin Yang, Keng Shen and Huifang Huang were involved in writing-review and editing.

Funding Supported by the Chinese Academy of Medical Sciences Initiative for Innovative Medicine (CAMS-2017-I2M-1-002).

Availability of data and material The dataset supporting this article is included within the article, and additional data are available from the corresponding author upon request.

\section{Declarations}

Conflict of interest The authors declare that they have no conflict of interest.

Ethical approval All procedures performed in the study were in accordance with the ethical standards of the institutional research committee and with the 1964 Helsinki declaration and its later amendments or comparable ethical standards.

Informed consent This research study was conducted retrospectively according to medical record data obtained during hospitalization. We consulted extensively with the IRB of Peking Union Medical College Hospital (PUMCH) who determined that our study did not require informed consent.

Consent to participate Not applicable.

Consent for publication Not applicable.

\section{References}

1. Dai CY, Lin CY, Tsai PC, Lin PY, Yeh ML, Huang CF et al (2018) Impact of tumor size on the prognosis of hepatocellular carcinoma in patients who underwent liver resection. J Chin Med Assoc 81(2):155-163

2. Meguro S, Yasuda M (2013) Alpha-fetoprotein-producing ovarian tumor in a postmenopausal woman with germ cell differentiation. Ann Diagn Pathol 17(1):140-144

3. Nogales FF, Bergeron C, Carvia RE, Alvaro T, Fulwood HR (1996) Ovarian endometrioid tumors with yolk sac tumor component, an unusual form of ovarian neoplasm. Analysis of six cases. Am J Surg Pathol. 20(9):1056-66
4. Tatarinov IS (1964) Detection of embryo-specific alpha-globulin in the blood serum of a patient with primary liver cancer. Vopr Med Khim 10:90-91

5. Norgaard-Pedersen B, Albrechtsen R, Teilum G (1975) Serum alpha-foetoprotein as a marker for endodermal sinus tumour (yolk sac tumour) or a vitelline component of "teratocarcinoma." Acta Pathol Microbiol Scand A 83(6):573-589

6. Li H, Xie Y, Shen Y (2019) Ovarian endometrioid adenocarcinoma a with yolk sac tumor in a 41-year-old woman: a case report. Int J Clin Exp Pathol 12(9):3549-3554

7. Kamoi S, Ohaki Y, Mori O, Okada S, Seto M, Matsushita N et al (2002) A case of ovarian endometrioid adenocarcinoma with yolk sac tumor component in a postmenopausal woman. APMIS 110(6):508-514

8. den Hollander MW, Westerink ND, Lubberts S, Bongaerts AH, Wolf RF, Altena R et al (2016) Bleomycin-induced pulmonary changes on restaging computed tomography scans in two thirds of testicular cancer patients show no correlation with fibrosis markers. Oncologist 21(8):995-1001

9. Bose CK, Mukherjea M (1993) Alpha-fetoprotein in advanced epithelial ovarian cancer. Br J Obstet Gynaecol 100(12):1149-1150

10. Casper S, van Nagell Jr JR, Powell DF, Dubilier LD, Donaldson ES, Hanson MB et al (1984) Immunohistochemical localization of tumor markers in epithelial ovarian cancer. Am J Obstet Gynecol 149(2):154-158

11. Abe A, Furumoto H, Yoshida K, Nishimura M, Irahara M, Kudo E et al (2008) A case of ovarian endometrioid adenocarcinoma with a yolk sac tumor component. Int J Gynecol Cancer 18(1):168-172

12. Lopez JM, Malpica A, Deavers MT, Ayala AG (2003) Ovarian yolk sac tumor associated with endometrioid carcinoma and mucinous cystadenoma of the ovary. Ann Diagn Pathol 7(5):300-305

13. Rutgers JL, Young RH, Scully RE (1987) Ovarian yolk sac tumor arising from an endometrioid carcinoma. Hum Pathol 18(12):1296-1299

14. Cao D, Guo S, Allan RW, Molberg KH, Peng Y (2009) SALL4 is a novel sensitive and specific marker of ovarian primitive germ cell tumors and is particularly useful in distinguishing yolk sac tumor from clear cell carcinoma. Am J Surg Pathol 33(6):894-904

15. Schuldt M, Rubio A, Preda O, Nogales FF (2016) GATA binding protein 3 expression is present in primitive patterns of yolk sac tumours but is not expressed by differentiated variants. Histopathology 68(4):613-615

16. Abiko K, Mandai M, Hamanishi J, Matsumura N, Baba T, Horiuchi A et al (2010) Oct4 expression in immature teratoma of the ovary: relevance to histologic grade and degree of differentiation. Am J Surg Pathol 34(12):1842-1848

17. Nonaka D, Chiriboga L, Soslow RA (2008) Expression of pax8 as a useful marker in distinguishing ovarian carcinomas from mammary carcinomas. Am J Surg Pathol 32(10):1566-1571

18. Nogales FF, Prat J, Schuldt M, Cruz-Viruel N, Kaur B, D’Angelo E et al (2018) Germ cell tumour growth patterns originating from clear cell carcinomas of the ovary and endometrium: a comparative immunohistochemical study favouring their origin from somatic stem cells. Histopathology 72(4):634-647

19. Cetin A, Bahat Z, Cilesiz P, Demirbag N, Yavuz E (2007) Ovarian clear cell adenocarcinoma producing alpha-fetoprotein: case report. Eur J Gynaecol Oncol 28(3):241-244

20. Kane SV, Wuntkal R, Ramadwar M, Tongaonkar HB (2004) Ovarian endometrioid carcinoma with yolk sac component in a young patient: a diagnostic and therapeutic dilemma. Aust N Z J Obstet Gynaecol 44(4):364-366

21. Horiuchi A, Osada R, Nakayama K, Toki T, Nikaido T, Fujii S (1998) Ovarian yolk sac tumor with endometrioid carcinoma arising from endometriosis in a postmenopausal woman, with special reference to expression of alpha-fetoprotein, sex steroid receptors, and p53. Gynecol Oncol 70(2):295-299 
22. Roth LM, Talerman A, Levy T, Sukmanov O, Czernobilsky B (2011) Ovarian yolk sac tumors in older women arising from epithelial ovarian tumors or with no detectable epithelial component. Int J Gynecol Pathol 30(5):442-451

23. Maida Y, Kyo S, Takakura M, Kanaya T, Inoue M (1998) Ovarian endometrioid adenocarcinoma with ectopic production of alphafetoprotein. Gynecol Oncol 71(1):133-136

24. Li M, Bao L, Cai H, Yang H, Ge W, Ren L et al (2014) Clinicopathologic features of ovarian clear cell carcinoma [correction of epithelial ovarian cancer] with yolk sac tumor component: report of a case. Zhonghua Bing Li Xue Za Zhi 43(2):127-128

25. Chao WT, Liu CH, Lai CR, Chen YJ, Chuang CM, Wang PH (2018) Alpha-fetoprotein-producing ovarian clear cell adenocarcinoma with fetal gut differentiation: a rare case report and literature review. J Ovarian Res 11(1):52

26. Morimoto A, Sudo T, Sakuma T, Yasuda M, Fujiwara K (2014) Alpha-fetoprotein-producing ovarian clear cell adenocarcinoma simulating fetal gut in a postmenopausal woman. Gynecol Oncol Case Rep 8:24-26

27. Takahashi Y, Mogami H, Hamada S, Urasaki K, Konishi I (2011) Alpha-fetoprotein producing ovarian clear cell carcinoma with a neometaplasia to hepatoid carcinoma arising from endometriosis: a case report. J Obstet Gynaecol Res 37(12):1842-1846
28. Konishi I, Fujii S, Kataoka N, Noda Y, Okamura H, Yamabe H et al (1988) Ovarian mucinous cystadenocarcinoma producing alpha-fetoprotein. Int J Gynecol Pathol 7(2):182-189

29. Nomura K, Miyasaka Y, Murae M, Terashima Y, Aizawa S (1992) Ovarian mucinous cystadenocarcinoma producing alpha-fetoprotein. A case report. Acta Pathol Jpn 42(5):372-375

30. Arai T, Kitayama Y, Koda K (1999) Ovarian mucinous cystadenocarcinoma with yolk sac tumor in a 71-year-old woman. Int J Gynecol Pathol 18(3):277-280

31. Suzuki T, Ino K, Kikkawa F, Shibata K, Kajiyama H, Morita T et al (2003) Cushing's syndrome due to ovarian serous adenocarcinoma secreting multiple endocrine substances: a case report and immunohistochemical analysis. Gynecol Oncol 90(3):662-666

32. Isonishi S, Ogura A, Kiyokawa T, Suzuki M, Kunito S, Hirama M et al (2009) Alpha-fetoprotein (AFP)-producing ovarian tumor in an elderly woman. Int J Clin Oncol 14(1):70-73

Publisher's Note Springer Nature remains neutral with regard to jurisdictional claims in published maps and institutional affiliations. 